\title{
Genesis and reversal of the ischemic phenotype in epithelial cells
}

\author{
Kevin T. Bush, Steven H. Keller, and Sanjay K. Nigam \\ Departments of Pediatrics and Medicine, University of California-San Diego, La Jolla, California, USA \\ Kevin T. Bush and Steven H. Keller contributed equally to this work. \\ Address correspondence to: Sanjay K. Nigam, University of California-San Diego, 9500 Gilman Drive (0693), \\ La Jolla, California 92093-0693, USA. Phone: (858) 822-3482; Fax: (858) 822-3483; E-mail: snigam@ucsd.edu.
}

Ischemic injury to predominantly epithelial organs such as the kidney remains a major cause of morbidity and mortality. For example, in hospitalized patients with acute renal failure, it is estimated that around $50 \%$ of all cases are ischemic in origin (1). Nevertheless, progress in the medical management of this and other syndromes in which ischemia occurs has been slow. In part, this may be related to our still rudimentary understanding of the cell biology underlying the ischemic epithelial phenotype and the molecular mechanisms behind the recovery of normal cell and tissue organization.

Epithelial tissues, such as the kidney and gut, not only provide a physical barrier between biologic compartments, but mediate vectorial and selective transport of ions, water, and macromolecules between blood and the external environment. These functions depend on the integrity of intercellular junctions (e.g., adherens, tight), the arrangement of lipids and proteins in the plasma membrane into strictly maintained apical and basolateral domains, and productive cell-substratum interactions, all of which are severely affected by ischemia/reperfusion.

Although other factors, such as oxidative damage and ion and $\mathrm{pH}$ changes, likely play important roles in the generation of the ischemic epithelial phenotype, much of the damage is believed to be due to depletion of cellular ATP $(2,3)$. Thus, cell culture models using agents that deplete cellular ATP have been used extensively to study ischemic injury in polarized epithelial cells (3). Although the fidelity with which these in vitro models reproduce the lesions observed in vivo has been debated, there is little doubt that these ATP depletion/repletion cell culture models provide valuable insights into the molecular mechanisms underlying ischemic injury and recovery, as similar cellular and molecular lesions are often found in cells of the ischemic whole organ. Many of these lesions are remarkably specific, biochemically definable, and potentially regulated; recovery from these lesions after short-term injury appears to be mediated by a combination of both previously elucidated and potentially novel sorting mechanisms that are transduced by "classical" signaling pathways.
Among other cellular and molecular lesions, ischemia and/or ATP depletion induces misfolding and/or aggregation of membrane and secreted proteins (4); disruption of the actin-based cytoskeleton (5); disturbances in apical-basolateral protein polarization (6); mislocalization and degradation of protein components of the intercellular junctions $(7,8)$; upregulation of a number of genes, including molecular chaperones $(4,9)$, growth factors and their receptors $(10)$; perturbation of integrin-mediated cell-substratum adhesion (11-13); and induction of programmed and nonprogrammed cell death (2). Alterations in the actin cytoskeleton and integrin-mediated cell-substratum interactions have been extensively reviewed elsewhere $(5,13)$. Here we focus primarily on recent information on lesions affecting the permeability barrier (intercellular junctions), signaling events involved in the recovery of this barrier, and the roles of molecular chaperones in protecting epithelial cells.

The establishment and maintenance of a selectively permeable barrier occur through homotypic interactions of the extracellular domains of multiple transmembrane adhesion molecules between adjacent cells. Examples of such proteins include E-cadherin in the adherens junction (AJ) and the occludin/claudin families in the tight junction (TJ). The intracellular domains of these adhesion molecules also interact (directly or indirectly) with a number of cytoplasmic proteins, including $\alpha, \beta$, and $\gamma$ catenin in the AJ, and zonula occludens-1 (ZO-1), ZO-2, ZO-3, and fodrin in the TJ, providing a functional link to the actin-based cytoskeleton. These interactions also modulate the stability of the adhesion proteins either by maintaining their appropriate conformations to recognize extracellular domains in adjoining cells or perhaps by inhibiting internalization and degradation. Under ischemic conditions, it appears that many of these cellular processes/structures are compromised, promoting junctional protein internalization and degradation, thereby disturbing the cell-cell interactions and the permeability barrier. Identifying molecular mechanisms underlying the cascade of events that induce cellular injury and those involved in the cell's recovery 
is key to developing rational therapeutic approaches to diminish the morbidity associated with ischemic injury to epithelial tissues.

\section{The adherens junction}

In cell culture models, polarization and intercellular junctions depend in large part on cell-cell contact mediated by E-cadherin and subsequent assembly of the AJ. For example, treatment of polarizing epithelial cells with anti-E-cadherin antibodies disrupts junction assembly and retards the generation of the polarized epithelial phenotype (14). Alternatively, transfection of E-cadherin into nonpolarized fibroblasts induces a polarized distribution of NaKATPase somewhat akin to that seen in polarized epithelial cells (15). In addition, the cadherin-catenin interactions within the $\mathrm{AJ}$ are also critical to the formation and maintenance of the polarized epithelia (16).

ATP depletion of cultured renal epithelial cells results in rapid internalization of E-cadherin (17). Even under normal physiological conditions, E-cadherin is selectively internalized and recycled to the cell surface in a clathrin-mediated recycling endosomal pathway (18); it remains to be determined whether this or another pathway is involved in internalization and re-sorting of E-cadherin after ischemia. A somewhat more prolonged insult leads not only to internalization of E-cadherin, but also to proteolytic clipping of this protein at a specific site and to the disruption of normal cadherin-catenin interactions (8). Identification of the site of E-cadherin cleavage as well as the protease involved will shed considerable mechanistic light on the disruption of the AJ in ischemia. Interestingly, although Ecadherin itself is cleaved, its cytoplasmic binding partners the catenins remain near their steady-state levels for prolonged periods of ATP depletion (8).

Because functional AJs are critical for the establishment and maintenance of tight polarized epithelia (including TJ formation and polarized sorting of membrane proteins), degradation of E-cadherin, as well as disruption of cadherin-catenin interactions, likely constitutes a critical lesion in epithelial ischemia. Over the long term, reassembly of the AJ in recovering epithelial tissue must depend on resynthesis of E-cadherin, assembly with the catenins, and re-formation of functional AJs. How this occurs remains unclear, although it is possible that the undegraded catenins are recruited from the cytoplasm and reassembled with de novo synthesized Ecadherin at the endoplasmic reticulum (ER) itself or at a more distal compartment in the secretory pathway, after which they may be targeted to the cell surface to help reconstruct the AJ. Repair of more permanent AJ structures might depend on turnover of the proteins exposed to ischemic injury and on the de novo synthesis and assembly of new components. As discussed later here, a limiting factor in the face of sustained ischemia may be the inability of the ER to fold newly synthesized membrane and secreted proteins such as E-cadherin (4).
The tight junction

The $\mathrm{TJ}$ is the most apically positioned junction and delineates the apical and basolateral surfaces of the epithelial cell. In addition, the TJ prevents lipid diffusion in the membrane between the apical to basolateral surfaces, and its component molecules form the physical basis for the permeability barrier to solutes and liquids. TJs are composed of transmembrane proteins, the occludins and claudins (19), which are probably linked to the cytoskeleton through interactions with cytoplasmic proteins, including the zonula occludens (ZO-1, ZO-2, and $\mathrm{ZO}-3$ ) and actin-binding proteins, such as fodrin (20).

In cell culture models of ischemia, occludin is internalized and becomes associated with large insoluble complexes of ZO-1 and fodrin (7). These junctional components appear to redistribute readily to their former locations after brief periods of ATP depletion and recovery in the presence of ATP. In contrast, prolonged and severe ATP depletion may, as with E-cadherin, target the junctional proteins into the cellular degradative pathway (K.T. Bush et al., unpublished observations); thus after prolonged injury, repair must take place by de novo synthesis together with movement of membrane proteins through the secretory pathway (also damaged by ischemic insult) and reassembly with cytosolic components. Where and how this occurs in the cell recovering from injury is a major question, as the sorting and bioassembly pathways may be distinct from those thought to operate under normal physiological conditions.

Although little is known at the biochemical or molecular level about reassembly of the TJ proteins after recovery from ischemia and/or ATP depletion, a great deal of work has been done on the biogenesis of the TJ using the Madin-Darby canine kidney (MDCK) cell "calcium switch" model for TJ assembly (20), aspects of which resemble cell culture models of ischemia. In this model, MDCK monolayers transferred to low calcium media lose cell-cell contacts and internalize their intercellular junctions. These cells also suffer a loss of apical and basolateral protein polarity, a disruption of their actin cytoskeletons, and a change in cell shape. These perturbations lead to disruption of vectorial transport and loss of the permeability barrier. Switching back to normal calcium media induces cell-cell contact and restores intercellular junctions, a normally configured cytoskeleton, and a more columnar cell shape; normal apical-basolateral polarity and barrier function are restored as well. Detailed studies of this model have implicated a number of signaling molecules in the reassembly of intercellular junctions, including protein kinase $C$, calcium, and heterotrimeric $G$ proteins (20). Although there are important distinctions in the cellular biochemistry between the calcium-switch and ATP depletion/repletion model (e.g., differential solubilities of junctional proteins), recent studies have also impli- 
cated signaling pathways involving intracellular calcium, small GTP-binding proteins and tyrosine kinase activities in recovery of the epithelial cell phenotype after short-term ATP depletion (21-23). It is not known exactly how these signaling events together influence $\mathrm{TJ}$ assembly, but indirect evidence suggests that certain signaling events modulate the rephosphorylation of TJ proteins, their release from cytoskeletal components, and perhaps dissolution of large macromolecular complexes and aggregates that accumulate during ATP depletion $(7,22,23)$. In addition, vesicular trafficking, endocytosis, and ubiquitination are all known to be modulated by cellular signaling, and they likely contribute to the protein processing involved in assembling and maintaining TJs.

\section{Cellular stress responses and cytoprotection}

Ischemic conditions, ATP depletion, or both are thought to promote the misfolding and/or denaturation of cellular proteins either directly or through perturbation of their biosynthetic/folding pathways ( 9 , 24). Such injury leads to a cellular stress response manifested by increases in the levels of mRNAs encoding the cytosolic stress proteins (e.g., the heat-shock proteins, including members of the Hsp70 family) (25), as well as the ER stress proteins (e.g., Grp78/BiP, Grp94, and ERp72) $(4,24)$. These two groups of stress proteins function as molecular chaperones in the folding and assembly of proteins by temporarily stabilizing polypeptides, preventing the occurrence of inappropriate intra- and intermolecular interactions and aggregation during the folding process (26); most appear to depend on cellular ATP for their function (27). In the stress response, molecular chaperones are thought to be essential to cell survival through their ability to bind abnormal proteins and prevent their aggregation.

Elevated levels of cytosolic chaperones, especially members of the Hsp70 family, correlate with enhanced survival of cells subjected to a subsequent injury including ischemia/reperfusion and energy deprivation (ATP depletion) (28). Although the exact mechanism of Hsp-mediated cytoprotection remains to be fully elucidated, it is possible that the chaperoning activity of the Hsp's protects cells by increasing protein refolding and limiting the potentially toxic aggregation of cellular proteins (29). In addition, increased levels of the Hsp's could protect cells after more prolonged ischemia/reperfusion and/or ATP depletion/repletion by interfering with NF- $\mathrm{BB}-$ mediated transcriptional activation of proinflammatory cytokine genes (30).

Similarly, ER molecular chaperones may have cytoprotective properties. For example, upregulation of both cytosolic and ER molecular chaperones after treatment with inhibitors of the proteasome has been shown to protect epithelial cells subjected to thermal stress (31). Evidence that ER chaperones alone can provide cytoprotection comes from experiments in which pretreatment with tunicamycin, an inhibitor of $\mathrm{N}$ linked glycosylation that specifically induces accumulation of ER molecular chaperones, was found to enhance the survival of ATP depleted renal epithelial cells in culture (9).

Thus, as in the case of the cytosolic heat-shock proteins, overexpression of the ER molecular chaperones correlates with increased survival of cells subjected to conditions modeling ischemia/reperfusion (9). As with Hsp70, the mechanism of cytoprotection remains unclear, although it is possible that enhanced cell survival is in part the result of increased chaperone function in the ER. Alternatively, as the ER serves as the major storage site of intracellular calcium and several of the ER molecular chaperones bind calcium, induction of these proteins may help moderate the dramatic rises in cytosolic free calcium that occurs in ischemia or ATP depletion and thus reduce the threat of oxidative stress to the cell (32-36).

\section{Molecular aspects of epithelial ischemia and recovery: outlines of a model}

To date, no central defect has been found that can account for the various aspects of the ischemic epithelial phenotype. Still, recent work has revealed the lesions of the ischemic epithelial cell to be remarkably specific. At least in cell culture models of ischemia, these lesions can be defined in considerable biochemical detail. Equally remarkable is the ability of the injured kidney, as well as injured cells in culture, to recover their structure and function virtually completely, even when considerably damaged by ischemia or ATP depletion. This recovery appears largely dependent on the magnitude of kidney ischemia and the duration of the insult. Thus, renal tubules injured by sublethal ischemic insult fully recover and re-establish kidney function. On the other hand, prolonged ischemia ultimately leads to cell death (necrosis and apoptosis) and can induce an inflammatory response that greatly limits the capacity of the tubules to recover. To understand better the molecular and cellular pathology of the ischemic epithelial phenotype and mechanisms underlying its restoration to normalcy, it is worthwhile to distinguish among events that lead to short-term and/or modest, intermediate, or prolonged and/or severe ischemic injury, as is shown in Figure 1. The model shown in this figure focuses primarily on damage to multiprotein complexes in intercellular junctions, such as the AJ. Similar consideration might apply to damage to other cell surface molecules and intracellular components.

Short-term ischemia results in the redistribution of cell-surface molecules and cytoskeletal disruption, but it does not induce detectable loss of E-cadherin or other rapidly degraded molecules. Under these conditions, recovery of the tight polarized epithelial cell phenotype is likely to depend on reusing existing compo- 
nents that became internalized, aggregated, or bound to the cytoskeleton during the ischemic period $(7,8$, 17). Based on work in cell culture models, this reassembly pathway probably depends on classical signaling pathways involving calcium (23), small GTP binding proteins (21), and tyrosine phosphorylation (22). This response may in fact be conceptually similar to the reassembly mechanisms elucidated using the MDCK calcium switch model, which depends solely on reuse of preexisting components. Hence, early intervention with drugs or growth factors that specifically modulate signaling through $\mathrm{IP}_{3}$-sensitive calcium stores, G-proteins, protein kinase $C$, and other kinases - all of which are implicated in the reassembly response during the calcium switch - may enhance recovery and minimize injury $(20,22)$. Nevertheless, it seems likely that some of the sorting and bioassembly pathways used by cells recovering from injury are distinct from those required under normal physiological conditions or in the calcium switch model. In this context, it is also worth noting that growth factor receptors may be internalized during ischemia, and the well-documented upregulation of growth factor receptors may be one response to this internalization (10). Facilitating the resorting of growth factor receptors to the cell surface through modulation of signaling pathways could enhance the effectiveness of endogenous and/or exogenous growth factors administered after ischemic insult.

During the intermediate stage of ischemia, some components of intercellular junctions (e.g., E-cadherin) and perhaps other proteins are rapidly degraded, whereas other components (e.g., ZO-1, catenins) remain intact (8), though, as in short-term ischemia, many of these proteins become redistributed at the plasma membrane, internalized, found tightly associated with the actin-based cytoskeleton, or aggregat-

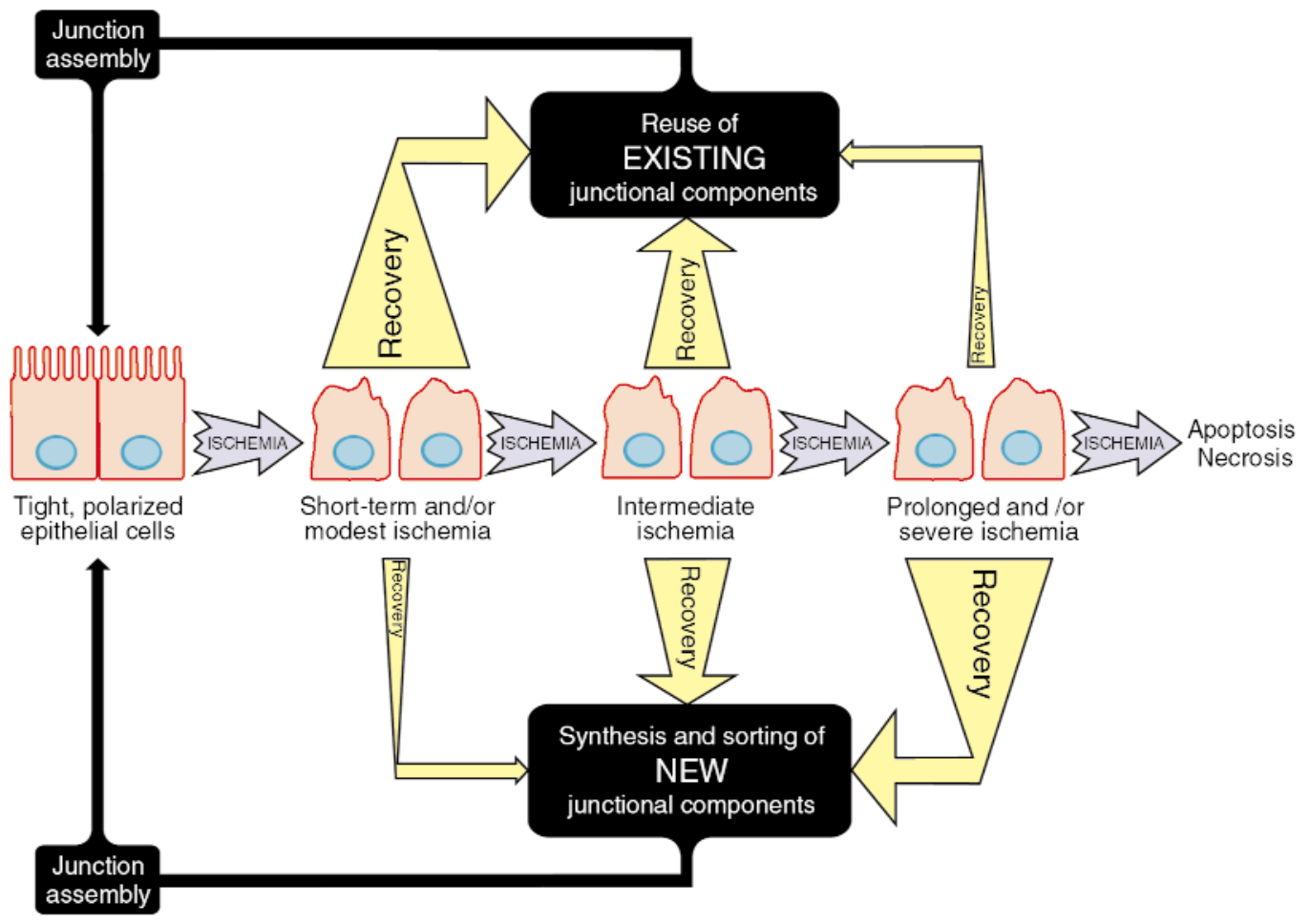

Figure 1

Model depicting general aspects of epithelial cell recovery after ischemia or ATP depletion. The ability of the cell to recover is dependent on the duration and extent of the ischemic insult and can be described as: (a) short-term and/or modest ischemia, (b) intermediate ischemia, and (c) prolonged and/or severe ischemia. After short-term and/or modest ischemia, degradation of critical junctional components has yet to occur, and cells can reestablish the tight, polarized epithelial cell phenotype primarily by reusing existing junctional components (e.g., E-cadherin, catenins) that have been internalized. As described in the text, this may require activation of signaling pathways involving tyrosine phosphorylation, calcium, and GTP. Intermediate ischemia is characterized by the beginning of the degradation of some of the junctional components (e.g., E-cadherin), and complete recovery from such an insult would likely involve a combination of reutilization of existing components together with synthesis of new junctional components. In the case of prolonged and/or severe ischemic injury, degradation of junctional components has proceeded to such an extent that recovery depends primarily on synthesis and assembly of new junctional macromolecular complexes, key proteins of which are folded in the ER. If the ischemic insult is not removed at this point, cell death (either apoptotic or necrotic) will ultimately be the result. The hypothesized relative importance of various pathways under each scenario is indicted by the thickness of the arrows. Intracellular junctions, such as the AJ, serve as an example, but other damaged cellular components may also become more dependent on de novo protein synthesis and ER folding/assembly for recovery as the length or severity of the ischemic insult increases. 
ed $(7,8)$. Even though the vast majority of proteins may not be rapidly degraded, it is conceivable that some are covalently modified in a way that limits their stability in the cell. Nevertheless, recovery would be expected to depend on reuse of existing components through the action of classical signaling events involving calcium, GTP, and tyrosine phosphorylation, together with de novo synthesis of key degraded proteins (e.g., E-cadherin) and reassembly of macromolecular complexes. Perhaps the rate-limiting step here would be assembly and folding within the endoplasmic reticulum, which itself is dysfunctional in the setting of ischemia $(4,24)$. Additional lesions may also exist elsewhere in the secretory pathway. This may require the cell to utilize novel sorting pathways not extensively used under normal physiological conditions. Furthermore, the final reassembly of multiprotein complexes such as those that constitute the $\mathrm{AJ}$ is likely to be quite different, as de novo synthesized Ecadherin that is translocated into the ER will presumably link to pre-existing catenins that were not degraded after injury but that have moved into an as yet unidentified cell compartment (in the case of the $\mathrm{TJ}$, its constituent proteins appear to associate with a cytoplasmic membrane compartment, and the cytoskeleton and may also aggregate) $(7,8)$. Depending on the duration and severity of ischemic injury, a combination of such potentially novel reassembly pathway(s) and the normal physiological secretory pathway (beginning with the biosynthesis and maturation of membrane proteins in the ER) may be necessary to effectively restore structures like the AJ, on which the cell's polarized distribution of membrane proteins and the tissue's capacity to act as a permeability barrier both depend.

After prolonged and severe, but still sublethal ischemic insult, it would be expected that many key membrane and secreted proteins (E-cadherin, claudins, occludins, integrins, matrix, molecules, and so forth) will be degraded or targeted for more rapid degradation. In addition, the injured cell, or its concerned neighbor, is likely to make an attempt at repair through the elaboration of growth factors and cytokines that must likewise pass through the secretory pathway. Therefore, a rate-limiting step for repair is likely to be bioassembly and folding in the ER and subsequent sorting through the secretory pathway (24). However in the setting of such severe ischemia, it is likely that the capacity of the ER to correct the misfolding/aggregation of secretory and membrane proteins through the action of ER molecular chaperones will be severely compromised (4). Some of the preexisting components that have not been degraded may still be useful, but the ultimate restoration of the polarized epithelial phenotype will require the biosynthesis and assembly of both secreted and cytosolic components of the crucial plasma membrane-associated complexes.
Hence, strategies designed to enhance epithelial cell recovery may have to target several distinct lesions. First, therapies should be designed to inhibit the internalization and promote the reuse of preexisting components, perhaps by targeting specific signaling events. Second, it will be necessary to inhibit the degradation of E-cadherin or other key proteins necessary for the maintenance of the polarized epithelial cell phenotype. Third, effective treatment, particularly of severe ischemic injury, may require enhancing the protein folding and assembly capacity in the ER and/or cytosol with agents which upregulate cytoprotective chaperones.

\section{Acknowledgments}

S.K. Nigam is supported in part by grants from the National Institute of Diabetes and Digestive and Kidney Diseases (RO1-DK53507 and RO1-DK51211). K.T. Bush is a recipient of a Scientist Development Award from the American Heart Association. S.H. Keller is supported in part by a Pilot Grant from the Cystic Fibrosis Foundation.

1. Thadhani, R., Pascual, M., and Bonventre, J.V. 1996. Acute renal failure. N. Engl. J. Med. 334:1448-1460.

2. Bonventre, J., et al. 1998. Acute renal failure. I. Relative importance of proximal vs. distal tubular injury. Am. J. Physiol. Renal Physiol. 275:F623-F631.

3. Molitoris, B., et al. 2000. Acute renal failure. II. Experimental models of acute renal failure: imperfect but indispensable. Am. J. Physiol. Renal Physiol. 278:F1-F12.

4. Kuznetsov, G., Bush, K.T., Zhang, P.L., and Nigam, S.K. 1996. Perturbations in maturation of secretory proteins and their association with endoplasmic reticulum chaperones in a cell culture model for epithelial ischemia. Proc. Natl. Acad. Sci. USA. 93:8584-8589.

5. Molitoris, B.A., Leiser, J., and Wagner, M.C. 1997. Role of the actin cytoskeleton in ischemia-induced cell injury and repair. Pediatr. Nephrol. 11:761-767.

6. Fish, E.M., and Molitoris, B.A. 1994. Alterations in epithelial polarity and the pathogenesis of disease states. N. Engl. J. Med. 330:1580-1588.

7. Tsukamoto, T., and Nigam, S.K. 1997. Tight junction proteins form large complexes and associate with the cytoskeleton in an ATP depletion model for reversible junction assembly. J. Biol. Chem. 272:16133-16139.

8. Bush, K.T., Tsukamoto, T., and Nigam, S.K. 2000. Selective degradation of E-cadherin and dissolution of E-cadherin-catenin complexes in epithelial ischemia. Am. J. Physiol. Renal Physiol. 278:F847-F852.

9. Bush, K.T., George, S.K., Zhang, P.L., and Nigam, S.K. 1999. Pretreatment with inducers of ER molecular chaperones protects epithelial cells subjected to ATP depletion. Am. J. Physiol. Renal Physiol. 277:F211-F218.

10. Hammerman, M., Safirstein, R., Harris, R., Toback, F., and Humes, H. 2000. Acute renal failure. III. The role of growth factors in the process of renal regeneration and repair. Am. J. Physiol. Renal Pbysiol. 279:F3-F11.

11. Gailit, J., Colflesh, D., Rabiner, I., Simone, J., and Goligorsky, M.S. 1993. Redistribution and dysfunction of integrins in cultured renal epithelial cells exposed to oxidative stress. Am. J. Physiol. Renal Physiol. 264:F149-F157.

12. Lieberthal, W., et al. 1997. Beta1 integrin-mediated adhesion between renal tubular cells after anoxic injury. J. Am. Soc. Nephrol. 8:175-183.

13. Zuk, A., Bonventre, J.V., Brown, D., and Matlin, K.S. 1998. Polarity, integrin, and extracellular matrix dynamics in the postischemic rat kidney. Am. J. Physiol. Renal Physiol. 275:C711-C731.

14. Gumbiner, B., Stevenson, B., and Grimaldi, A. 1988. The role of the cell adhesion molecule uvomorulin in the formation and maintenance of the epithelial junctional complex. J. Cell Biol. 107:1575-1587.

15. McNeill, H., Ozawa, M., Kemler, R., and Nelson, W.J. 1990. Novel function of the cell adhesion molecule uvomorulin as an inducer of cell surface polarity. Cell. 62:309-316.

16. Steinberg, M.S., and McNutt, P.M. 1999. Cadherins and their connections: adhesion junctions have broader functions. Curr. Opin. Cell Biol. 11:554-560. 
17. Mandel, L.J., Doctor, R.B., and Bacallao, R. 1994. ATP depletion: a novel method to study junctional properties in epithelial tissues. II. Internalization of $\mathrm{Na}^{+}, \mathrm{K}(+)$-ATPase and E-cadherin. J. Cell Sci. 107:3315-3324.

18. Le, T.L., Yap, A.S., and Stow, J.L. 1999. Recycling of E-cadherin: a potential mechanism for regulating cadherin dynamics. J. Cell Biol. 146:219-232.

19. Tsukita, S., Furuse, M., and Itoh, M. 1999. Structural and signalling molecules come together at tight junctions. Curr. Opin. Cell Biol. 11:628-633.

20. Denker, B.M., and Nigam, S.K. 1998. Molecular structure and assembly of the tight junction. Am. J. Physiol. Renal Physiol. 274:F1-F9.

21. Gopalakrishnan, S., Raman, N., Atkinson, S.J., and Marrs, J.A. 1998. Rho GTPase signaling regulates tight junction assembly and protects tight junctions during ATP depletion. Am.J. Physiol. Cell. Physiol. 275:C798-C809.

22. Tsukamoto, T., and Nigam, S.K. 1999. Role of tyrosine phosphorylation in the reassembly of occludin and other tight junction proteins. Am.J. Physiol. Renal Physiol. 276:F737-F750.

23. Ye, J., Tsukamoto, T., Sun, A., and Nigam, S.K. 1999. A role for intracellular calcium in tight junction reassembly after ATP depletion-repletion. Am. J. Physiol. Renal Physiol. 277:F524-F532.

24. Kuznetsov, G., and Nigam, S.K. 1998. Folding of secretory and membrane proteins. N. Engl. J. Med. 339:1688-1695.

25. Van Why, S.K., et al. 1999. Thresholds for cellular disruption and activation of the stress response in renal epithelia. Am. J. Physiol. Renal Physiol. 277:F227-F234.

26. Gething, M.J., and Sambrook, J. 1992. Protein folding in the cell. Nature. 355:33-45.

27. Nigam, S.K., et al. 1994. A set of endoplasmic reticulum proteins possessing properties of molecular chaperones includes $\mathrm{Ca}(2+)$-binding proteins and members of the thioredoxin superfamily. J. Biol. Chem.
269:1744-1749

28. Gabai, V.L., and Kabakov, A.E. 1993. Rise in heat-shock protein level confers tolerance to energy deprivation. FEBS Lett. 327:247-250.

29. Georgopoulos, C., and Welch, W.J. 1993. Role of the major heat shock proteins as molecular chaperones. Annu. Rev. Cell Biol. 9:601-634.

30. Yoo, C.G., et al. 2000. Anti-inflammatory effect of heat shock protein induction is related to stabilization of I kappa B alpha through preventing I kappa B kinase activation in respiratory epithelial cells. $J$. Immunol. 164:5416-5423.

31. Bush, K.T., Goldberg, A.L., and Nigam, S.K. 1997. Proteasome inhibition leads to a heat-shock response, induction of endoplasmic reticulum chaperones, and thermotolerance. J. Biol. Chem. 272:9086-9092.

32. Dong, Z., Saikumar, P., Griess, G.A., Weinberg, J.M., and Venkatachalam, M.A. 1998. Intracellular Ca2+ thresholds that determine survival or death of energy-deprived cells. Am. J. Pathol. 152:231-240.

33. Kribben, A., et al. 1994. Evidence for role of cytosolic free calcium in hypoxia-induced proximal tubule injury. J. Clin. Invest. 93:1922-1929.

34. Liu, H., Miller, E., van de Water, B., and Stevens, J.L. 1998. Endoplasmic reticulum stress proteins block oxidant-induced $\mathrm{Ca} 2+$ increases and cell death. J. Biol. Chem. 273:12858-12862.

35. Yu, Z., Luo, H., Fu, W., and Mattson, M.P. 1999. The endoplasmic reticulum stress-responsive protein GRP78 protects neurons against excitotoxicity and apoptosis: suppression of oxidative stress and stabilization of calcium homeostasis. Exp. Neurol. 155:302-314.

36. Bian, X., Hughes, F.M., Jr., Huang, Y., Cidlowski, J.A., and Putney, J.W., Jr. 1997. Roles of cytoplasmic Ca2+ and intracellular Ca2+ stores in induction and suppression of apoptosis in S49 cells. Am. J. Physiol. Cell. Physiol. 272:C1241-C1249. 